\title{
Study of Electric charge induction with a three-dimensional model using the finite elements method
}

\author{
Alexandre de Resende Camara*1,200, Zeila Virginia Torres Santos ${ }^{3}$, Clara Johanna Pacheco ${ }^{4}$, Marcos \\ Vinicius Colaço Gonçalves ${ }^{1}$ \\ ${ }^{1}$ Universidad do Estado de Rio de Janeiro, Instituto de Física, Rio de Janeiro, RJ, Brasil \\ ${ }^{2}$ Universidad do Estado de Rio de Janeiro, Departamento de Engenharia, Programa de Graduação em Engenharia Eletrônica, \\ Rio de Janeiro, RJ, Brasil \\ ${ }^{3}$ Universidad de Ciencias y Humanidades, Centro de Investigación Interdisciplinar Ciencia y Sociedad, Los Olivos, Lima, Perú \\ ${ }^{4}$ Universidade Federal do Rio de Janeiro, Laboratório de Ensaios Não Destrutivos, Corrosão e Soldagem, Rio de Janeiro, RJ, \\ Brasil
}

Received on May 16, 2018; Revised on June 20, 2018; Accepted on June 22, 2018.

\begin{abstract}
One of the first subjects studied in Electricity and Magnetism classes is how one can make an electrically neutral object be an electrically charged object. There are three ways to do it: charging by friction, charging by contact and charging by induction. Induction charging is a method used to charge an object without actually touching the object to any other charged object. In order to illustrate the explanation of how the process of charging an electrically neutral body by induction, a 3D model was built using the finite elements method to show the increasing of the surface charge density in a initially neutral sphere when the distance between this sphere and a negatively charged bar decreases.
\end{abstract}

Keywords: Induction of charges, FEM, Comsol Multiphysycs ${ }^{\circledR}$.

\section{Introduction}

In the beginning of courses as Engineering and Physics, the students have the first contact with the Electromagnetic Theory. As the first subject studied, we have the explanation of the electric charge that is the fundamental element of the Electromagnetism. The electric charge in the particles describes the gain or loss of electrons that is an intrinsic subatomic property. Therefore, there is a correlation between electric and magnetic fields. We can ask ourselves, what keeps the matter together? The answer comes from the Forces that govern the Universe, being in the case of our question the Electromagnetic Forces and the Strong Nuclear Force [1].

In a general case, all bodies are called electrically neutral, i. e., the amount of positive and negative charges distributed in the bodies are the same[2]. However, there are three different ways to charge an electrically neutral body: charging by friction, by contact and by induction. In the first case, for instance, when we brush our hair, they exchange electrons by friction and after that if we approximate the brush to some small pieces of paper, they will be attracted. After the charging by friction process, the bodies have charges with opposite signals but with the same intensity.

*Correspondence email address: professoralexandrecamara@gmail.com
In the second case, if we take an object that is previously charged (positively or negatively) and touch it to another object that can either also be previously charged or electrically neutral, the charges will be redistributed in the whole system (body $1+$ body 2 ). When they are separated, the final electric charge of each object will have the same signal and the individual charge will be, in this particular case, half of the sum of the initial charges.

Finally, what happens if we bring a charged object close to a conductive surface? In this case as the charged object is closer to the conductive surface, as higher is the amount of charges with opposite signal that appear in the surface of the conductive material. We know this process as charging by induction.

We show in Figure 1 a neutral sphere and a positive charged bar. When the electrically charged bar is approached towards a neutral conductive sphere, the electric force, given by Coulomb's Law, makes the positive charges of the sphere be repulsed by the positive charges of the bar and it also makes the negative charges of the sphere be attracted by the positive charges of the bar [3]. Even though the charges of the sphere are separated, i.e., they are not uniformly distributed in the surface of the sphere, the total electric charge of the sphere is still zero.

In order to complete the induction process, it is necessary neutralize the excess of positive charges repulsed by the charged bar. To do that, we need to connect the 
sphere to the ground, after the separation of the protons and electrons, as shown in Figure 2(a). Once the sphere is connected to the ground, electrons migrates towards the sphere to neutralize the repulsed positive charges by the charged bar. After that, as shown in Figure 2(b), with the electric bar still near to the sphere, the ground connection is removed and the electron that entered the sphere remains there. The last step of the charging by induction process is to move the charged bar away to the sphere. In Figure 2(c), it is possible to see that, when the bar is removed, there is an excess of negative charges on the sphere. So, the sphere that was initially neutral, now has electric charges with opposite signal to the charged bar. The final value of the charge of the sphere depends on the distance between the sphere and the bar.

In order to improve the understanding of this process, we built a three-dimensional model to simulate the charging of a neutral sphere by induction.

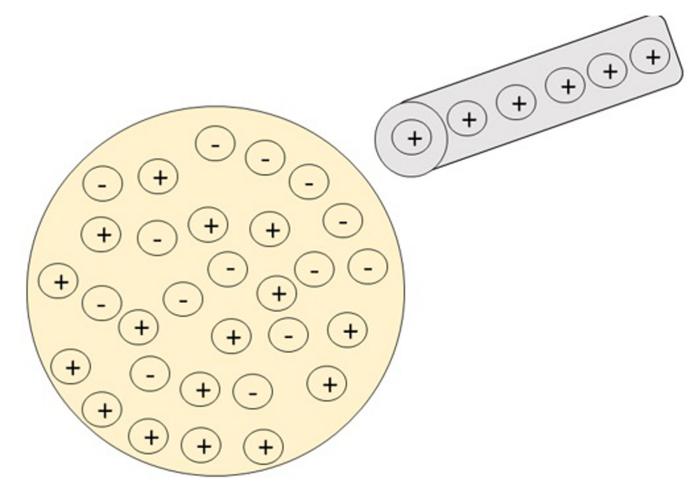

Figure 1: A positive charged bar is placed close to an electrically neutral conductive sphere. The electrons begin to travel in the direction of the bar by electric attraction, and the positive charges are repulsed in the direction of the opposite side of the sphere.

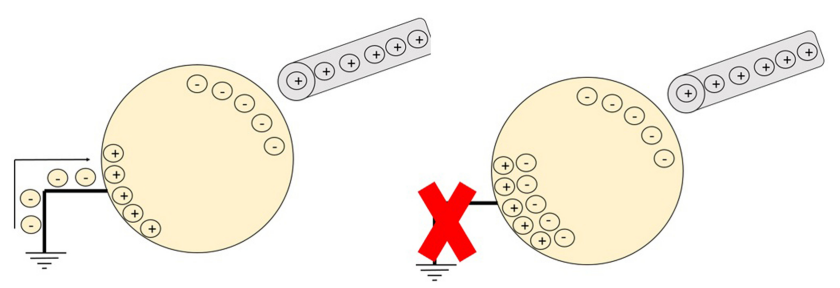

(a)

(b)

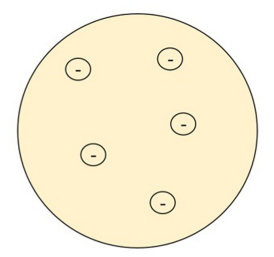

(c)

Figure 2: The effect of the ground connection of the sphere. (a) The electrons in the ground migrates to the sphere in order to neutralize the excess of positive charges repulsed by the charged bar.

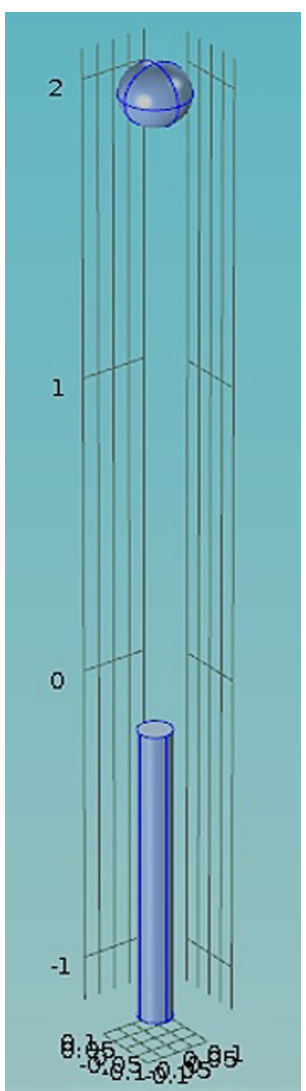

Figure 3: Geometry drawn in Comsol Multiphysics to study the electric charge induction problem.

Herein Comsol Multiphysics ${ }^{\circledR}$ software is used to simulate quoted phenomenon. The software is a comprehensive simulation environment for a wide array of applications, yet structured and user-friendly for all kind of users.

The aim of this work is the use of simulation as didactic resource to demonstrate physical phenomenon both for high school and undergraduate student.

\section{Method}

Using the Comsol Multiphysics ${ }^{\circledR}$ software [4], we built a model to illustrate the explanation about the induction of electric charges on objects. The software solves many mathematical problems related to Chemistry, Engineering and Physics using the Finite Elements Method. Herein, Comsol Multiphysics was used to solve the problem of the induction of positive charges in the surface of an initially electrically neutral sphere when the distance between it and a negatively charged bar decreases (Figure 3).

Figure 3 shows an electrically neutral glass sphere that is initially located $2 \mathrm{~m}$ far from a negative charged cylindrical copper bar. The sphere has radius equal to $0.1 \mathrm{~m}$ and the bar is a $0.05 \mathrm{~m}$ width, $1 \mathrm{~m}$ height cylinder. The physics of the numerical model here is based on the COMSOL Multiphysics solution electrostatic problem 
assuming that the glass sphere is under $0 \mathrm{~V}$ and the copper bar us under $-1000 \mathrm{~V}$. The equations that the software will solve are:

$$
\begin{gathered}
\vec{\nabla} \bullet \vec{D}=\rho \\
\vec{\nabla} V=\vec{E}
\end{gathered}
$$

where in equation 1 , which is the differential form of the Gauss Law, $\vec{D}$ is the electric displacement field and $\rho$ is the surface charge density and in equation $2, \mathrm{~V}$ is the electric potential and $\vec{E}$ is the conservative electric field.

The last step is the creation of the mesh to solve the electrostatic equations. It is needed to split the geometry of sphere and bar in many pieces using a mesh. Each peace is an element and the model will solve the equations 1 and 2 for each element. So, in this case, a highly dense three-dimensional mesh is established over whole bar and sphere, as shown in Figure 4.

\section{Results and Discussions}

After solve the electric potential equation, Comsol Multiphysics shows the results of the simulations in a $3 \mathrm{D}$ color map. Figure 5(a)-5(d) show the surface charge density in the glass sphere in different distances from the charged bar.

As expected, the surface charge density in the sphere increases if the distance between the bar and the sphere decreases. Furthermore, it is possible to see that the amount of charges in the sphere is not uniformly distributed, which confirm the dependence of the attraction force among the negative charges of the bar and the positive charges of the sphere on the distance. In Fig 5(a), the sphere is placed $2 \mathrm{~m}$ far from the charged bar. Even though it is a considerable distance, there is a positive surface charge density in the hemisphere closer to the bar (and, consequently, a negative surfce charge density if the analysis was made in the hemisphere more distant from the bar). In this case, $\rho_{2 \mathrm{~m}}=56.5 \mathrm{nC} / \mathrm{m}^{2}$. When the distance between the sphere and the bar descreases, it is possible to observe that the surface charge density increased. Fig. 5(b) shows the sphere $1 \mathrm{~m}$ far from the bar. Although the distance is half of the distance in the previous case, the value of the surface charge density slightly increased $\left(\rho_{1 \mathrm{~m}}=62 \mathrm{nC} / \mathrm{m}^{2}\right)$. This result shows that the increasing of the value of the induced surface charge density does not depends linearly with the distance between the bodies. Reducing again by a factor 2

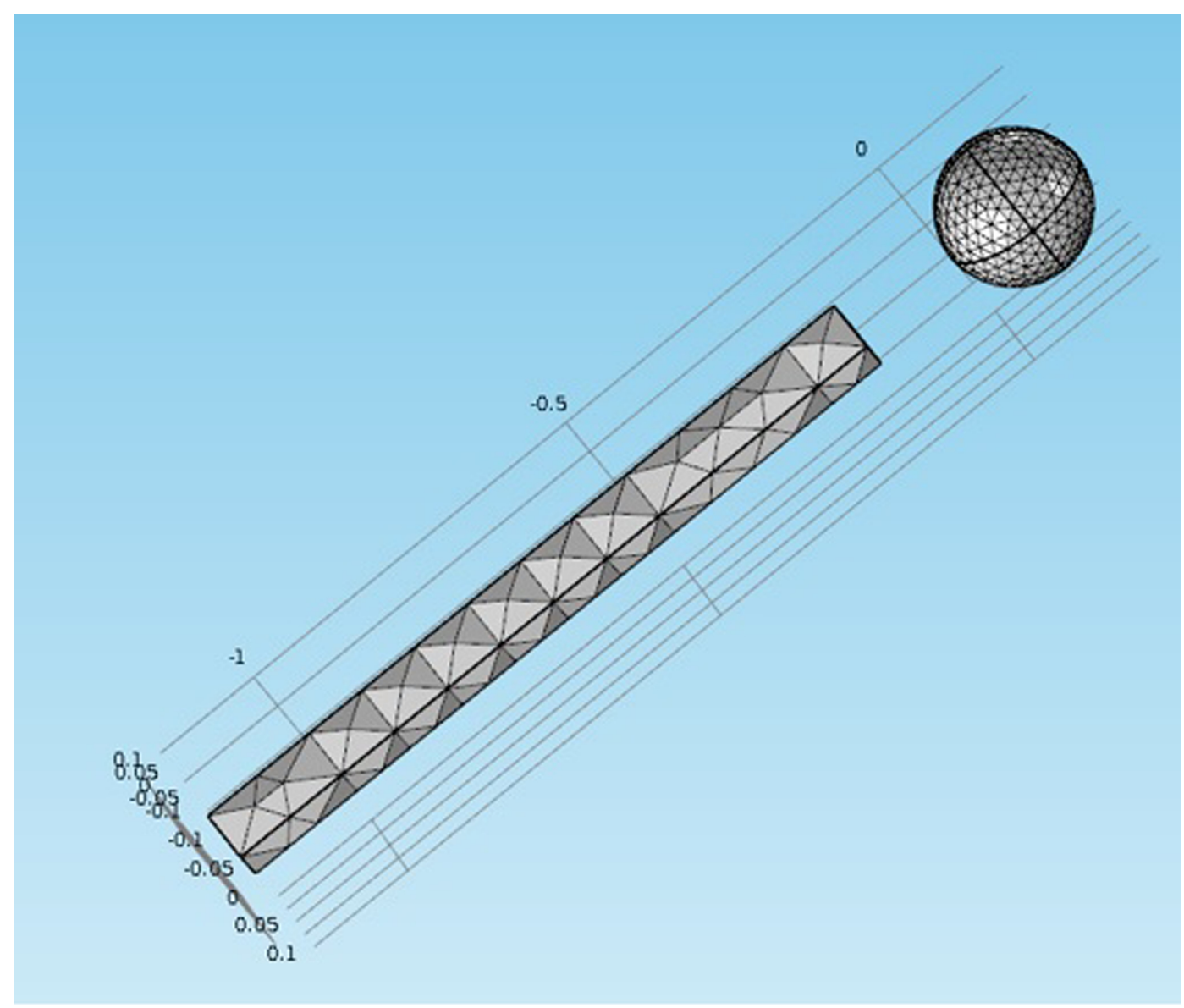

Figure 4: Example of the mesh created in the sphere and in the bar to solve the Gauss Law and the electric potential equation. The mesh size varies from $1 \mathrm{~mm}$ to $10 \mathrm{~cm}$. The software automatically defines the size of each element in the geometry. 


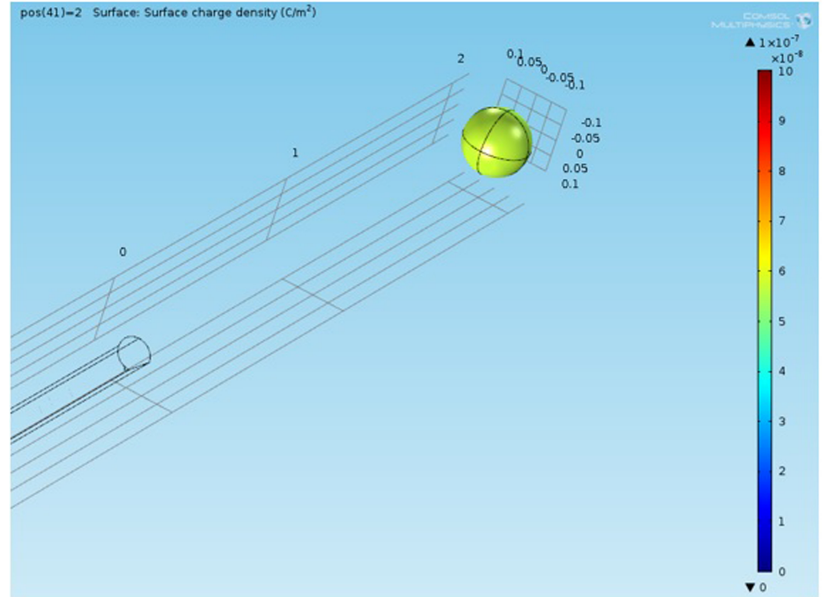

(a)

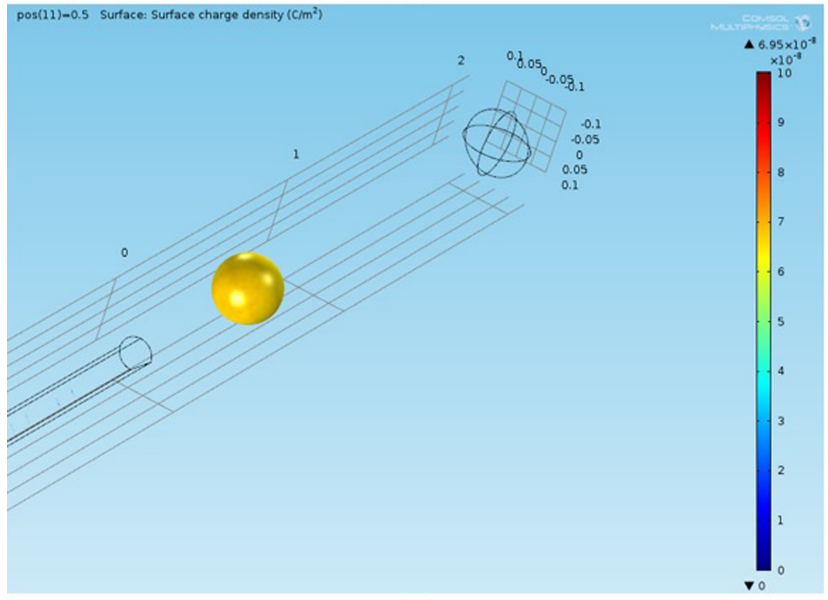

(c)

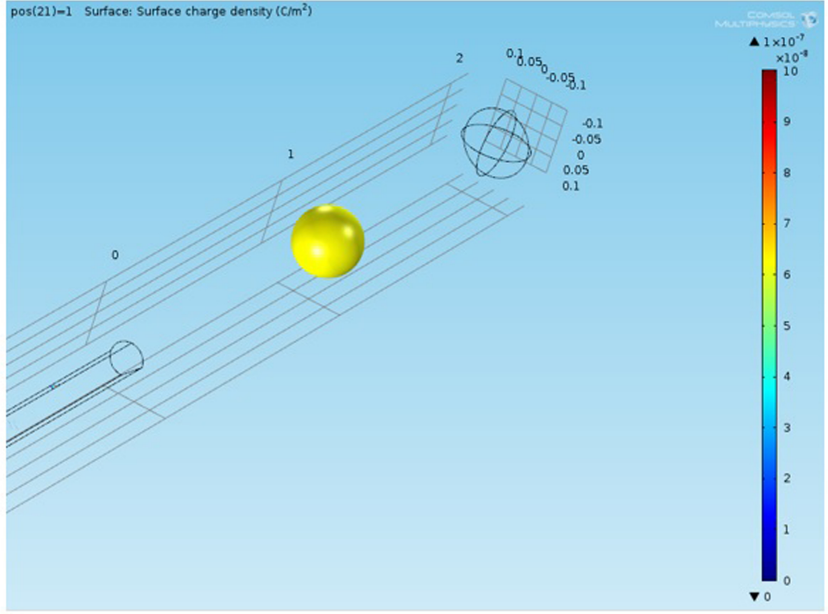

(b)

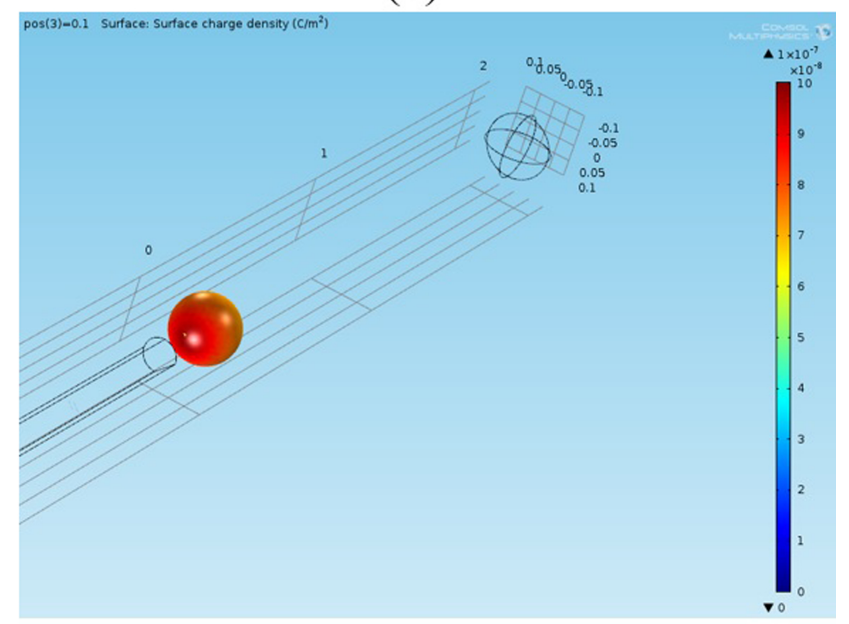

(d)

Figure 5: 3D color maps that shows the surface density charge in the glass sphere when the distance between sphere and charged bar is $(\mathrm{a}) \mathrm{d}=2 \mathrm{~m}\left(\rho=5.65 \times 10^{-8} \mathrm{C} / \mathrm{m}^{2}\right) ;(\mathrm{b}) \mathrm{d}=1 \mathrm{~m}\left(\rho=6.20 \times 10^{-8} \mathrm{C} / \mathrm{m}^{2}\right) ;(\mathrm{c}) \mathrm{d}=0.5 \mathrm{~m}\left(\rho=6.64 \times 10^{-8} \mathrm{C} / \mathrm{m}^{2}\right)$ and $(\mathrm{d})$ $\mathrm{d}=0.1 \mathrm{~m}\left(\rho=8.19 \times 10^{-8} \mathrm{C} / \mathrm{m}^{2}\right)$.

the distance between sphere and bar (Fig. 5(c)), it is possible to observe that the increasing of the surface charge density is still small $\left(\rho_{0.5 \mathrm{~m}}=66.4 \mathrm{nC} / \mathrm{m}^{2}\right)$. However, reducing the distance between sphere and bar to $0.1 \mathrm{~m}$, the surface charge density experiences a large increase of its value. Now, as shown in Figure 5(d), the sphere is shown in a strong red, and $\rho_{0.1 \mathrm{~m}}=81.9 \mathrm{nC} / \mathrm{m}^{2}$. In order to illustrate the behavior of the surface charge density with the distance between sphere and bar, a 2D graphic was made and it is shown in Fig. 6.

As seen in Fig. 6, the surface charge density experiences a large increase of its value when sphere and bar are very close. In the case of this model it occurs to distances smaller than $0.25 \mathrm{~m}$. As expected, the induced surface charge goes to zero if the separation distance is large enough.

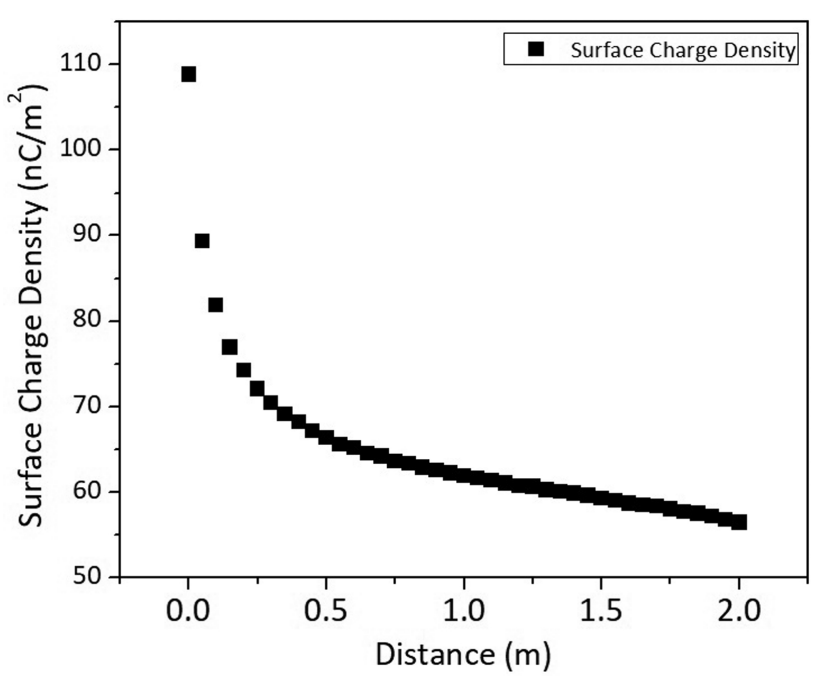

Figure 6: Graphic showing the behavior of the surface charge density with the distance. 


\section{Conclusions}

In this work, we showed a $3 \mathrm{D}$ model to illustrate the electric charge induction problem using the finite element method. All results are given in colored maps, which improve the understanding of what happens to a neutral sphere when it is placed close to a charged bar. This model can be used in the practice classes in order to preview the results of experiments, e. g., the charge induction in an electroscope by a glass rod that is a very common experiment in teaching laboratories.

\section{Acknowledgments}

The authors want to thank Dr. Isabel Carvalho by the use of Comsol Multiphysics.

\section{References}

[1] J.D. Jackson, Classical Electrodynamics (Wiley, New York, 1975), $3^{\text {rd }}$ ed.

[2] K.D. Machado, Eletromagnetismo (Toda Palavra, Ponta Grossa, 2012), v. 1, $1^{\text {st }}$ ed.

[3] A. Osaru, Electrical Study, available in https: //electricalstudy.sarutech.com/static-electricfield/index.html

[4] https://www.comsol.com/ 